COMMUNICATIONS IN

ANALYSIS AND GEOMETRY

Volume 11, Number 4, 737-750, 2003

\title{
The Dirac-Witten Operator on Spacelike Hypersurfaces
}

\begin{abstract}
Oussama Hijazi And XiaO Zhang
We establish optimal lower bounds for the eigenvalues of the DiracWitten operator of compact (with or without boundary) spacelike hypersurfaces of a Lorentzian manifold, whose metric satisfies the Einstein field equations and whose energy momentum-tensor satisfies the dominant energy condition.
\end{abstract}

\section{Introduction.}

The well-known spinorial proof of the positive mass theorem given by E. Witten [W] is based on a subtle use of the Weitzenböck type formula for the hypersurface Dirac-type operator called the Dirac-Witten operator. The mass is given by the limit at infinity of some boundary integral term.

In the past two decades, lower bounds for the eigenvalues of the classical Dirac operator on closed Riemannian spin manifolds were intensively studied (see for example, [F, Hi1]). Manifolds with minimal eigenvalues are characterized by the existence of solutions of some overdetermined systems as for Killing spinors.

More recently [HMZ], under some natural boundary conditions, such results were extended to the case of Riemannian compact spin manifolds with boundary. Moreover, in [Z3, HZ] (see also [M]) examined the corresponding questions to the Dirac-Witten operator when the ambient manifold is Riemmanian and spin.

In this paper, we consider compact (with or without boundary) spacelike hypersurfaces of an $(n+1)$-dimensional Lorentzian manifold, whose metric

\footnotetext{
${ }^{1}$ The authors would like to thank Helga Baum, Xavier Charuel, Nicolas Ginoux and Bertrand Morel for pointing out the existence, in all dimensions, on a spacelike hypersurface of a positive definite Hermitian scalar product.

${ }^{2}$ Research of X.Z. is partially supported by CNSF \# 2009100 and CAS \# 2278400. This work is partially done during his visit to the Institut Élie Cartan, Université Henri Poincaré, Nancy 1, and he would like to thank the Institute for its hospitality.
} 
satisfies the Einstein field equations and whose energy momentum-tensor satisfies the dominant energy condition. In [B], Helga Baum showed that the spinor bundle of any spacelike hypersurface could be endowed with a positive definite Hermitian scalar product. In terms of this scalar product, we are able to prove a basic lower bound for the square of the eigenvalues of the Dirac-Witten operator in terms of the energy-momentum tensor of the ambient Lorentzian space (see inequality (5)). We then improve this result by introducing the spinorial energy-momentum tensor (see inequality (9)). The limiting-cases are also studied.

A generalized conformal Laplacian $L$ is then defined (see (12)) on the spacelike hypersurface. With the help of a classical conformal argument, under the assumption of the existence of a positive eigenfunction of $L$ in the set of regular conformal transformations, it is then shown that, up to a dimensional constant, the first eigenvalue of $L$ is less than the first eigenvalues of the Dirac-Witten operator (see (13) and (14)).

In the last section, as in [HMZ], the case of compact spacelike hypersurfaces with boundary is examined. Under some natural local boundary conditions (16), the previous results are then extended to (19), (21), (24) and (25). As an application, we show that an eigenvalue lower bound of the Dirac-Witten operator provides a criterion on the nonexistence of black holes in general relativity (see Corollary 1 ).

\section{The Dirac-Witten operator.}

Let $\left(N^{n, 1}, \widetilde{g}\right)$ be an $(n+1)$-dimensional Lorentzian manifold, and $\widetilde{g}$ be a Lorentzian metric of signature $(-1,1, \cdots, 1)$ which satisfies the Einstein field equations

$$
\widetilde{R i c}-\frac{1}{2} \widetilde{R} \widetilde{g}=T
$$

where $\widetilde{R i c}, \widetilde{R}$ are Ricci curvature tensor, scalar curvature of $\widetilde{g}$ respectively, and $T$ is the energy-momentum tensor. Let $(M, g)$ be a spacelike spin hypersurface of $N$ with the induced Riemannian metric $g$ and timelike unit normal vector $e_{0}$. We say that $(N, \widetilde{g})$ satisfies the dominant energy condition if for each timelike vector $e_{0}, T\left(e_{0}, e_{0}\right) \geq 0$ and $T\left(e_{0}, \cdot\right)$ is a non-spacelike covector. If $M$ is a spacelike hypersurface with orthonormal basis $\left\{e_{\alpha}\right\}$ and its dual basis $\left\{e^{\alpha}\right\}$ such that $e_{0}$ is normal to $M$ and $\left\{e_{i}\right\}$ is tangent to $M$, then the dominant energy condition implies that

$$
T_{00} \geq \sqrt{\sum_{i} T_{0 i}^{2}} .
$$


Denote by $\mathbb{S}$ the (local) spinor bundle of $N$. Since $M$ is spin, $\mathbb{S}$ exists globally over $M$. This spinor bundle $\mathbb{S}$ is called the hypersurface spinor bundle of $M$. If $\widetilde{\nabla}$ and $\nabla$ denote respectively the Levi-Civita connections of $\widetilde{g}$ and $g$, the same symbols are used to denote their lift to the hypersurface spinor bundle.

According to $[\mathrm{B}]$, there exists a Hermitian inner product $($,$) on \mathbb{S}$ over $M$ which is compatible with the spin connection $\widetilde{\nabla}$. For any covector $\widetilde{X}$ of $N$, and hypersurface spinors $\phi, \psi$, we have

$$
(\widetilde{X} \cdot \phi, \psi)=(\phi, \widetilde{X} \cdot \psi),
$$

where "." denotes the Clifford multiplication. Note that this inner product is not positive definite. Moreover, there exists on $\mathbb{S}$ over $M$ a positive definite Hermitian inner product defined by

$$
\langle,\rangle=\left(e^{0} \cdot,\right) \text {. }
$$

Obviously,

$$
\left\langle e^{0} \cdot \phi, \psi\right\rangle=\left\langle\phi, e^{0} \cdot \psi\right\rangle,
$$

and for any covector $X$ of $M$,

$$
\langle X \cdot \phi, \psi\rangle=-\langle\phi, X \cdot \psi\rangle .
$$

Fix a point $p \in M$ and an orthonormal basis $\left\{e_{\alpha}\right\}$ of $T_{p} N$ with $e_{0}$ normal and $e_{i}$ tangent to $M$ such that $\left(\nabla_{i} e_{j}\right)_{p}=0$ and $\left(\widetilde{\nabla}_{0} e_{j}\right)_{p}=0$. Let $\left\{e^{\alpha}\right\}$ be the dual basis. Then $\left(\widetilde{\nabla}_{i} e^{j}\right)_{p}=-h_{i j} e^{0},\left(\widetilde{\nabla}_{i} e^{0}\right)_{p}=-h_{i j} e^{j}$ where $h_{i j}=\left\langle\widetilde{\nabla}_{i} e_{0}, e_{j}\right\rangle$, $1 \leq i, j \leq n$, are the components of the second fundamental form at $p$. We have

$$
\widetilde{\nabla}_{i}=\nabla_{i}-\frac{1}{2} h_{i j} e^{0} \cdot e^{j} \cdot
$$

This fact implies that the spinor connection $\nabla$ is compatible with the positive definite inner product $\langle$,$\rangle . Moreover,$

$$
\nabla_{i}\left(e^{0} \cdot \phi\right)=e^{0} \cdot \nabla_{i} \phi .
$$

with the inner product $\langle$,$\rangle except when h_{i j}=0$, for all $i, j$. Its formal self-adjoint with respect to $\langle$,$\rangle is nothing but$

$$
\widetilde{\nabla}_{i}^{*}=-\nabla_{i}+h_{i j} e^{0} \cdot e^{j} \cdot .
$$

The Dirac-Witten operator over $M$ is defined by

$$
\widetilde{D}=\sum_{i} e^{i} \cdot \widetilde{\nabla}_{i}
$$


This operator $\widetilde{D}$ is formally self-adjoint with respect to $\langle$,$\rangle and its square$ is given by the following Weitzenböck type formula

$$
\widetilde{D}^{2}=\widetilde{\nabla}^{*} \widetilde{\nabla}+\frac{1}{2}\left(T_{00}+T_{0 i} e^{0} \cdot e^{i} \cdot\right) .
$$

Here, and henceforth, the Einstein summation notation is used. For any real function $u$ on $N$, consider the conformal metric $\overline{\widetilde{g}}=e^{2 u} \widetilde{g}$. Denote by $G_{u}$ the isometry between the associated $\mathrm{SO}_{n}$-principal bundles $\mathrm{SO}_{\widetilde{g}} N$ and $\mathrm{SO}_{\bar{\sigma}} N$. The isometry $G_{u}$ induces an isometry between the $\operatorname{Spin}_{n}$-principal bundles, $\operatorname{Spin}_{\tilde{g}} N$ and $\operatorname{Spin}_{\overline{\tilde{g}}} N$ as well as an isometry between their hypersurface spinor bundles $\mathbb{S}$ and $\overline{\mathbb{S}}\left(\equiv G_{u} \mathbb{S}\right)$.

For any sections $\phi, \psi$ of the spinor bundle $\mathbb{S}$, we denote by $\bar{\phi}=G_{u} \phi$, and $\bar{\psi}=G_{u} \psi$ the corresponding sections of $\overline{\mathbb{S}}$. Then

$$
(\phi, \psi)_{\widetilde{g}}=(\bar{\phi}, \bar{\psi})_{\bar{g}}, \quad\langle\phi, \psi\rangle_{\widetilde{g}}=\langle\bar{\phi}, \bar{\psi}\rangle_{\bar{g}}
$$

and the Clifford multiplication on $\overline{\mathbb{S}}$ is given by

$$
\overline{e^{\alpha}}-\bar{\phi}=\overline{e^{\alpha} \cdot \phi}
$$

Denote by $M_{t}$ the tubular neighborhood of $M$ obtained by taking the parallel transport of $e_{0}$. Note that the restriction of $\overline{\widetilde{g}}$ to $M$ gives rise to a conformal change of metric on $M$. Denote the regular conformal class of $N$ by

$$
\mathcal{U}=\left\{u \in C^{\infty}(N),\left.d u\left(e_{0}\right)\right|_{M_{t}}=0\right\} .
$$

With respect to the regular conformal class, if $\mathcal{U}$ is nonempty, similar to [HZ], the conformal transformation of the Dirac-Witten operator is

$$
\overline{\widetilde{D}}\left(e^{-\frac{(n-1)}{2} u} \bar{\phi}\right)=e^{-\frac{(n+1)}{2} u} \overline{\widetilde{D} \phi}
$$

Lemma 1. Let $\left(N^{n, 1}, \widetilde{g}\right)$ be an $(n+1)$-dimensional Lorentzian manifold which satisfies the Einstein field equations (1), and let $M$ be a spacelike spin hypersurface. Denote by $\left\{e_{\alpha}\right\}$ a local orthonormal basis of $N$ and $\left\{e^{\alpha}\right\}$ its dual basis where $e_{0}$ is normal to $M$ and $\left\{e_{i}\right\}$ are tangent to $M$. If there exist a nontrivial hypersurface spinor $\phi$, and real functions $t, t_{i}$ with

$$
t|\phi|^{2}+\left\langle\phi, t_{i} e^{0} \cdot e^{i} \cdot \phi\right\rangle=0, \quad \text { and } \quad t \geq \sqrt{\sum_{i} t_{i}^{2}},
$$

then there exists a real function $f$ such that

$$
t=|f||\phi|^{2}, \quad \text { and } \quad t_{i}=f\left\langle\phi, e^{0} \cdot e^{i} \cdot \phi\right\rangle .
$$


Proof : Define the one-form $\delta$ by $\delta:=\left\langle\phi, e^{0} \cdot e^{i} \cdot \phi\right\rangle e^{i}$. Obviously,

$$
|\delta|^{2}=\sum_{i}\left\langle\phi, e^{0} \cdot e^{i} \cdot \phi\right\rangle^{2}
$$

Moreover,

$$
\left\langle e^{0} \cdot \phi, \delta \cdot \phi\right\rangle=\sum_{i}\left\langle\phi, e^{0} \cdot e^{i} \cdot \phi\right\rangle^{2}
$$

Since $\left\langle e^{0} \cdot \phi, \delta \cdot \phi\right\rangle \leq|\delta||\phi|^{2}$, it follows

$$
\sum_{i}\left\langle\phi, e^{0} \cdot e^{i} \cdot \phi\right\rangle^{2} \leq|\phi|^{4}
$$

Now we have

$$
t|\phi|^{2}=-t_{i}\left\langle\phi, e^{0} \cdot e^{i} \cdot \phi\right\rangle \leq \sqrt{\sum_{i} t_{i}^{2}} \sqrt{\sum_{i}\left\langle\phi, e^{0} \cdot e^{i} \cdot \phi\right\rangle^{2}} \leq t|\phi|^{2} .
$$

So all the inequalities involved are in fact equalities.

Q.E.D.

\section{Hypersurfaces without boundary.}

Now we estimate the lower bounds of nonzero eigenvalues of the DiracWitten operator for spacelike spin hypersurfaces $(M, g)$ without boundary. The Weitzenböck type formula (3) gives

$$
\int_{M}|\widetilde{D} \phi|^{2}=\int_{M}|\widetilde{\nabla} \phi|^{2}+\frac{1}{2}\left\langle\phi,\left(T_{00}+T_{0 i} e^{0} \cdot e^{i} \cdot\right) \phi\right\rangle .
$$

Under the dominant energy condition (2), identity (4) and Lemma 1 imply that any nonzero eigenvalue of the Dirac-Witten operator of a compact spacelike spin hypersurface should satisfy

$$
\lambda^{2}>\frac{1}{2} \inf _{M}\left(T_{00}-\sqrt{\sum_{i} T_{0 i}^{2}}\right) .
$$

We now apply Friedrich's argument $[\mathrm{F}]$ to improve this lower bound.

Theorem 1. Let $\left(N^{n, 1}, \widetilde{g}\right)$ be an $(n+1)$-dimensional Lorentzian manifold which satisfies the Einstein field equations (1) and the dominant energy condition (2). Denote by $M$ a compact spacelike spin hypersurface without 
boundary and by $\left\{e_{\alpha}\right\}$ a local orthonormal basis of $N$. Let $\left\{e^{\alpha}\right\}$ be its dual basis such that $e_{0}$ is normal to $M$ and $\left\{e_{i}\right\}$ are tangent to $M$. Let $\lambda$ be a nonzero eigenvalue of the Dirac-Witten operator associated with an eigenspinor $\phi$. Then

$$
\lambda^{2} \geq \frac{n}{2(n-1)} \inf _{M}\left(T_{00}-\sqrt{\sum_{i} T_{0 i}^{2}}\right) .
$$

If $\lambda^{2}$ achieves equality in (5), then there exists a real function $f$ such that

$$
T_{00}=\frac{2(n-1)}{n} \lambda^{2}+|f||\phi|^{2}, \quad \text { and } \quad T_{0 i}=f\left\langle\phi, e^{0} \cdot e^{i} \cdot \phi\right\rangle .
$$

Proof : Define a modified spinor connection $\widehat{\nabla}^{\lambda}$ by $\widehat{\nabla}_{i}^{\lambda}=\widetilde{\nabla}_{i}+\frac{\lambda}{n} e^{i}$. Then for any spinorfield $\phi$ one has

$$
\left|\widehat{\nabla}^{\lambda} \phi\right|^{2}=|\widetilde{\nabla} \phi|^{2}-\frac{\lambda^{2}}{n}|\phi|^{2}
$$

which when substituted in (4), yields

$$
\int_{M}\left|\widehat{\nabla}^{\lambda} \phi\right|^{2}=\int_{M} \frac{n-1}{n} \lambda^{2}|\phi|^{2}-\frac{1}{2}\left\langle\phi,\left(T_{00}+T_{0 i} e^{0} \cdot e^{i} \cdot\right) \phi\right\rangle .
$$

This implies (5). If $\lambda^{2}$ achieves equality in (5), then

$$
\widetilde{\nabla}_{i} \phi=-\frac{\lambda}{n} e^{i} \cdot \phi, \quad \text { and } \quad \lambda^{2}=\frac{n}{2(n-1)}\left(T_{00}-\sqrt{\sum_{i} T_{0 i}^{2}}\right) .
$$

Taking the derivative once more, it follows

$$
\widetilde{\nabla}_{i} \widetilde{\nabla}_{j} \phi=-\frac{\lambda}{n} \widetilde{\nabla}_{i} e^{j} \cdot \phi+\frac{\lambda^{2}}{n^{2}} e^{j} \cdot e^{i} \cdot \phi .
$$

Thus

$$
\begin{aligned}
\left(T_{00}+T_{0 i} e^{0} \cdot e^{i} \cdot\right) \phi & =e^{i} \cdot e^{j} \cdot\left(\widetilde{\nabla}_{i} \widetilde{\nabla}_{j}-\widetilde{\nabla}_{j} \widetilde{\nabla}_{i}\right) \phi \\
& =\frac{\lambda^{2}}{n^{2}} e^{i} \cdot e^{j} \cdot\left(e^{j} \cdot e^{i} \cdot-e^{i} \cdot e^{j} \cdot\right) \phi \\
& =\frac{2(n-1)}{n} \lambda^{2} \phi .
\end{aligned}
$$

It is then sufficient to apply Lemma 1, from which we deduce (6). Q.E.D. 
Now we use the spinor energy-momentum tensor and some conformal arguments to estimate the nonzero eigenvalues of the Dirac operator [Hi1, Hi3]. For any spinorfield $\phi$, define the associated spinor energy-momentum 2-tensor $Q_{\phi}$ on the complement of its zero set by,

$$
Q_{\phi, i j}=\frac{1}{2} \operatorname{Re}\left(e^{i} \cdot \widetilde{\nabla}_{j} \phi+e^{j} \cdot \widetilde{\nabla}_{i} \phi, \phi /|\phi|^{2}\right) .
$$

If $\phi$ is an eigenspinor of the Dirac-Witten operator $\widetilde{D}$, the tensor $Q_{\phi}$ is welldefined in the sense of distribution and its trace is the the corresponding eigenvalue.

Theorem 2. Under the same conditions as Theorem 1, if $\lambda$ is a nonzero eigenvalue of the Dirac-Witten operator associated with an eigenspinor $\phi$, then

$$
\lambda^{2} \geq \frac{1}{2} \inf _{M}\left(T_{00}-\sqrt{\sum_{i} T_{0 i}^{2}}+2\left|Q_{\phi}\right|^{2}\right),
$$

where $\left|Q_{\phi}\right|^{2}=\sum_{i, j} Q_{\phi, i j}^{2}$. If $\lambda^{2}$ achieves equality in (9), then there exists a real function $f$ such that

$$
T_{00}=2\left(\lambda^{2}-\left|Q_{\phi}\right|^{2}\right)+|f||\phi|^{2}, \quad \text { and } \quad T_{0 i}=f\left\langle\phi, e^{0} \cdot e^{i} \cdot \phi\right\rangle .
$$

Proof : Define a modified spinor connection $\widehat{\nabla}^{Q}$ by

$$
\widehat{\nabla}_{i}^{Q}=\widetilde{\nabla}_{i}+Q_{\phi, i j} e^{j} \cdot
$$

If $\widetilde{D} \phi=\lambda \phi$, then it is straightforward to see that

$$
\left|\widehat{\nabla}^{Q} \phi\right|^{2}=|\widetilde{\nabla} \phi|^{2}-\left|Q_{\phi}\right|^{2}
$$

which together with (4), yield

$$
\int_{M}\left|\widehat{\nabla}^{Q} \phi\right|^{2}=\int_{M} \lambda^{2}|\phi|^{2}-\frac{1}{2}\left\langle\phi,\left(T_{00}+T_{0 i} e^{0} \cdot e^{i} \cdot\right) \phi\right\rangle-\left|Q_{\phi}\right|^{2} .
$$

This implies (9). If $\lambda^{2}$ achieves equality in (9), then

$$
\widetilde{\nabla}_{i} \phi=-Q_{\phi, i j} e^{j} \cdot \phi, \quad \text { and } \quad \lambda^{2}=\frac{1}{2}\left(T_{00}-\sqrt{\sum_{i} T_{0 i}^{2}}\right)+\left|Q_{\phi}\right|^{2} .
$$

Second derivatives of the eigenspinor $\phi$ are then given by

$$
\widetilde{\nabla}_{i} \widetilde{\nabla}_{j} \phi=-\nabla_{i} Q_{\phi, j k} e^{k} \cdot \phi-Q_{\phi, j k} e^{k} \cdot \nabla_{i} \phi+\frac{1}{2} h_{i a} Q_{\phi, j k} e^{0} \cdot e^{a} \cdot e^{k} \cdot \phi .
$$


Thus

$$
\begin{aligned}
\left(T_{00}+T_{0 i} e^{0} \cdot e^{i} \cdot\right) \phi= & e^{i} \cdot e^{j} \cdot\left(\widetilde{\nabla}_{i} \widetilde{\nabla}_{j}-\widetilde{\nabla}_{j} \widetilde{\nabla}_{i}\right) \phi \\
= & -\left(\nabla_{i} Q_{\phi, j k}+\nabla_{j} Q_{\phi, i k}\right) e^{i} \cdot e^{j} \cdot e^{k} \cdot \phi \\
& +Q_{\phi, i l} Q_{\phi, j k} e^{i} \cdot e^{j} \cdot\left(e^{k} \cdot e^{l} \cdot-e^{l} \cdot e^{k} \cdot\right) \phi \\
& +\frac{1}{2}\left(-h_{i k} Q_{\phi, j k}+h_{j k} Q_{\phi, i k}\right) e^{i} \cdot e^{j} \cdot e^{0} \cdot \phi \\
= & -\nabla_{i} Q_{\phi, i k} e^{k} \cdot \phi+2\left(\lambda^{2}-\left|Q_{\phi}\right|^{2}\right) \phi \\
& +\frac{1}{2}\left(-h_{i k} Q_{\phi, j k}+h_{j k} Q_{\phi, i k}\right) e^{i} \cdot e^{j} \cdot e^{0} \cdot \phi .
\end{aligned}
$$

Since $e^{k}$ and $e^{i} \cdot e^{j} \cdot e^{0} \cdot(i \neq j)$ are anti-symmetric with respect to the inner product $\langle$,$\rangle , we deduce that$

$$
\left(T_{00}-2\left(\lambda^{2}-\left|Q_{\phi}\right|^{2}\right)\right)|\phi|^{2}+\left\langle\phi, T_{0 i} e^{0} \cdot e^{i} \cdot \phi\right\rangle=0 .
$$

Again we apply Lemma 1 to deduce (10).

Q.E.D.

Remark 1. By the Cauchy-Schwarz inequality we have

$$
n\left|Q_{\phi}\right|^{2} \geq\left(\operatorname{Tr} Q_{\phi}\right)^{2}=\lambda^{2}
$$

Hence (5) follows from (9).

Let $\widetilde{\triangle}, \triangle$ be the positive Laplace operators of $N$ and $M$ respectively. If $\overline{\widetilde{g}}=e^{2 u} \widetilde{g}$, then the corresponding Ricci and scalar curvatures are related by

$$
\begin{aligned}
\widetilde{\widetilde{R}}_{\alpha \beta}= & \widetilde{R}_{\alpha \beta}-(n-1) \widetilde{\nabla}_{\alpha} \widetilde{\nabla}_{\beta} u+(n-1) \widetilde{\nabla}_{\alpha} u \widetilde{\nabla}_{\beta} u \\
& -\left(-\widetilde{\triangle} u+(n-1)|\widetilde{\nabla} u|^{2}\right) \widetilde{g}_{\alpha \beta} \\
\widetilde{\widetilde{R}}= & e^{-2 u}\left(\widetilde{R}+2 n \widetilde{\triangle} u-n(n-1)|\widetilde{\nabla} u|^{2}\right)
\end{aligned}
$$

If $\mathcal{U}$ is nonempty, take $u \in \mathcal{U}$ and let $U>0$, with $U^{\frac{4}{n-2}}=e^{2 u}$, then over $M$, one has

$$
\begin{aligned}
& \bar{T}_{00}=\overline{\widetilde{R}}_{00}-\frac{1}{2} \overline{\widetilde{R}} \overline{\widetilde{g}}_{00}=T_{00}+\frac{2(n-1)}{n-2} U^{-1} \triangle U \\
& \bar{T}_{0 i}=\overline{\widetilde{R}}_{0 i}=\widetilde{R}_{0 i}=T_{0 i} .
\end{aligned}
$$

Define the generalized conformal Laplacian on $M$ by

$$
L=\frac{4(n-1)}{n-2} \triangle+2\left(T_{00}-\sqrt{\sum_{i} T_{0 i}^{2}}\right) .
$$


(If $h_{i j}=0$, then $L$ is exactly the classical conformal Laplacian.)

Theorem 3. Under the same conditions as Theorem 1, let $\lambda$ be a nonzero eigenvalue of the Dirac-Witten operator associated with an eigenspinor $\phi$. If $\mathcal{U}$ is nonempty and there is a positive eigenfunction $U$ of $L$ such that $U^{\frac{4}{n-2}}=e^{2 u}$ for some $u \in \mathcal{U}$, then

$$
\begin{gathered}
\lambda^{2} \geq \frac{n}{4(n-1)} \mu_{1}, \\
\lambda^{2} \geq \frac{1}{4} \mu_{1}+\inf _{M}\left|Q_{\phi}\right|^{2},
\end{gathered}
$$

where $\mu_{1}$ is the first eigenvalue of $L$.

Proof : Take the regular conformal transformation $\overline{\widetilde{g}}=e^{2 u} \widetilde{g}$ with $e^{2 u}=$ $U_{\mu_{1}}^{\frac{4}{n-2}}$, where $U_{\mu_{1}}$ is the corresponding positive eigenfunction of $L$. Note that for a regular conformal transformation, we have $\bar{T}_{\overline{0} \overline{0}}=e^{-2 u} T_{00}, \bar{T}_{\overline{0} \bar{i}}=$ $e^{-2 u} T_{0 i}$, and $\bar{Q}_{\bar{\phi}, \bar{i} \bar{j}}=e^{-u} Q_{\phi, i, j}$. Identities (7) and (11) written wrt this new metric $\overline{\widetilde{g}}$, yield

$$
\begin{aligned}
\int_{M}\left|\overline{\widehat{\nabla}^{\lambda}} \bar{\psi}\right|_{\bar{g}}^{2} v_{\bar{g}}= & \int_{M} e^{-u}\left[\left(\frac{n-1}{n} \lambda^{2}+\frac{n-1}{n-2} U_{\mu_{1}}^{-1} \triangle U_{\mu_{1}}\right)|\phi|^{2}\right. \\
& \left.-\frac{1}{2}\left\langle\phi,\left(T_{00}+T_{0 i} e^{0} \cdot e^{i} \cdot\right) \phi\right\rangle\right] v_{g} \\
\leq & \int_{M} e^{-u}\left(\frac{n-1}{n} \lambda^{2}-\frac{1}{4} U_{\mu_{1}}^{-1} L U_{\mu_{1}}\right)|\phi|^{2} v_{g}, \\
\int_{M}\left|\overline{\widehat{\nabla}^{Q}} \bar{\psi}\right|_{\bar{g}}^{2} v_{\bar{g}}= & \int_{M} e^{-u}\left[\left(\lambda^{2}-\left|Q_{\phi}\right|^{2}+\frac{n-1}{n-2} U_{\mu_{1}}^{-1} \triangle U_{\mu_{1}}\right)|\phi|^{2}\right. \\
& \left.-\frac{1}{2}\left\langle\phi,\left(T_{00}+T_{0 i} e^{0} \cdot e^{i} \cdot\right) \phi\right\rangle\right) v_{g} \\
\leq & \int_{M} e^{-u}\left(\lambda^{2}-\left|Q_{\phi}\right|^{2}-\frac{1}{4} U_{\mu_{1}}^{-1} L U_{\mu_{1}}\right)|\phi|^{2} v_{g},
\end{aligned}
$$

where $\psi=e^{-\frac{n-1}{2} u} \phi$. Hence (13) and (14).

Q.E.D.

\section{Hypersurfaces with boundary.}

We now assume that $M$ has boundary $\partial M$ endowed with its induced Riemannian and spin structures. Let $\nabla^{\partial M}$ be the Levi-Civita connection of $\partial M$ and denote by the same symbol their corresponding lift to the spinor bundle $\mathbb{S}$. Fix a point $q \in \partial M$ and an orthonormal basis of $T_{q} M$ with $e_{r}$ the 
outward normal to $\partial M$ and $e_{a}$ tangent to $\partial M$ such that for $1 \leq a, b \leq n-1$, $\left(\nabla_{a}^{\partial M} e_{b}\right)_{q}=0,\left(\nabla_{e_{r}} e_{b}\right)_{q}=0$. Let $\left\{e^{r}, e^{a}\right\}$ be the dual coframe. Then, $\left(\nabla_{a} e^{b}\right)_{q}=-h_{a b}^{\partial M} e^{r},\left(\nabla_{a} e^{r}\right)_{q}=h_{a b}^{\partial M} e^{b}$, where $h_{a b}^{\partial M}=\left\langle\nabla_{a} e_{r}, e_{b}\right\rangle$ are the components of the second fundamental form at $q$, and we have

$$
\nabla_{a}=\nabla_{a}^{\partial M}+\frac{1}{2} h_{a b}^{\partial M} e^{r} \cdot e^{b} \cdot
$$

Let $H^{\partial M}=\sum h_{a a}^{\partial M}$ be the unnormalized mean curvature of $M$ (In the above notation, the standard sphere $S_{r}^{2}=\partial B_{r}^{3}$ has positive mean curvature $H=\frac{3}{r}$ ). Denote the (intrinsic) Dirac operator of the boundary $\partial M$ acting on $\mathbb{S}$ by $D^{\partial M}=e^{a} \cdot \nabla_{a}^{\partial M}$. Then $e_{r} \cdot D^{\partial M}$ is also compatible with the metric $\langle$,$\rangle . Moreover, \nabla_{a}^{\partial M}\left(e^{r} \cdot \phi\right)=e^{r} \cdot \nabla_{a}^{\partial M} \phi$, and $D^{\partial M}\left(e^{r} \cdot \phi\right)=-e^{r} \cdot D^{\partial M} \phi$, see [HMZ]. In the boundary case, the Weitzenböck type formula (3) translates to

$$
\begin{aligned}
\int_{M}|\widetilde{D} \phi|^{2}= & \int_{M}|\widetilde{\nabla} \phi|^{2}+\frac{1}{2}\left\langle\phi,\left(T_{00}+T_{0 i} e^{0} \cdot e^{i} \cdot\right) \phi\right\rangle \\
& -\int_{\partial M}\left\langle\phi, e^{r} \cdot e^{a} \cdot \widetilde{\nabla}_{a} \phi\right\rangle * e^{r}
\end{aligned}
$$

where "*" is the star operator of $M$. Since

$$
\widetilde{\nabla}_{a}=\nabla_{a}^{\partial M}+\frac{1}{2} h_{a b}^{\partial M} e^{r} \cdot e^{b} \cdot-\frac{1}{2} h_{a j} e^{0} \cdot e^{j} \cdot
$$

we finally obtain

$$
\begin{aligned}
\int_{M}|\widetilde{D} \phi|^{2}= & \int_{M}|\widetilde{\nabla} \phi|^{2}+\frac{1}{2}\left\langle\phi,\left(T_{00}+T_{0 i} e^{0} \cdot e^{i} \cdot\right) \phi\right\rangle \\
& -\int_{\partial M}\left\langle\phi, e^{r} \cdot D^{\partial M} \phi\right\rangle * e^{r} \\
& -\frac{1}{2} \int_{\partial M}\left\langle\phi,\left(H^{\partial M}-\operatorname{tr}\left(\left.h\right|_{\partial M}\right) e^{r} \cdot e^{0} \cdot\right) \phi\right\rangle * e^{r} \\
& +\frac{1}{2} \int_{\partial M}\left\langle\phi, h_{a r} e^{0} \cdot e^{a} \cdot \phi\right\rangle * e^{r} .
\end{aligned}
$$

There is the following well-known local boundary condition on $\partial M$ :

$$
\phi= \pm e^{r} \cdot e^{0} \cdot \phi
$$

which ensures that $\widetilde{D}$ is formally self-adjoint. Note that (15) together with (16) were used to prove the positive mass theorem for black holes by Gibbons, 
Hawking, Horowitz and Perry, see [GHHP, He2]. Under this local boundary condition, (15) becomes

$$
\begin{aligned}
\int_{M}|\widetilde{D} \phi|^{2}= & \int_{M}|\widetilde{\nabla} \phi|^{2}+\frac{1}{2}\left\langle\phi,\left(T_{00}+T_{0 i} e^{0} \cdot e^{i} \cdot\right) \phi\right\rangle \\
& +\frac{1}{2} \int_{\partial M}\left( \pm \operatorname{Tr}\left(\left.h\right|_{\partial M}\right)-H^{\partial M}\right)|\phi|^{2} * e^{r} .
\end{aligned}
$$

Recall that the boundary $\partial M$ is a future or past apparent horizon if the condition

$$
\pm \operatorname{Tr}\left(\left.h\right|_{\partial M}\right)-H^{\partial M} \geq 0
$$

holds on $\partial M$.

Theorem 4. Under the same conditions as Theorem 1, let $\lambda$ be a nonzero eigenvalue of the Dirac-Witten operator associated with an eigenspinor $\phi$. If $M$ has boundary $\partial M$ which is a future or past apparent horizon, then under the local boundary condition (16), we have

$$
\lambda^{2} \geq \frac{n}{2(n-1)} \inf _{M}\left(T_{00}-\sqrt{\sum_{i} T_{0 i}^{2}}\right) .
$$

If $\lambda^{2}$ achieves equality in (19), then there exists a real function $f$ such that

$$
T_{00}=\frac{2(n-1)}{n} \lambda^{2}+|f||\phi|^{2}, \quad \text { and } \quad T_{0 i}=f\left\langle\phi, e^{0} \cdot e^{i} \cdot \phi\right\rangle .
$$

Moreover, $\pm \operatorname{Tr}\left(\left.h\right|_{\partial M}\right)=H^{\partial M}$ on $\partial M$.

Corollary 1. Under the same conditions as Theorem 1, and $M$ has boundary $\partial M$. If there is a constant $C>0$ such that

$$
T_{00}-\sqrt{\sum_{i} T_{0 i}^{2}}>C,
$$

and there exists a spinor $\phi$ which satisfies the local boundary condition (16) and $\widetilde{D} \phi=\lambda \phi$ for some constant $\lambda \leq \frac{n}{2(n-1)} C$, then $\partial M$ is neither a future nor a past apparent horizon.

Theorem 5. Under the same conditions as Theorem 1, let $\lambda$ be a nonzero eigenvalue of the Dirac-Witten operator associated with an eigenspinor $\phi$. If $M$ has boundary $\partial M$ which is a future or past apparent horizon, then under the local boundary condition (16), we have

$$
\lambda^{2} \geq \frac{1}{2} \inf _{M}\left(T_{00}-\sqrt{\sum_{i} T_{0 i}^{2}}+2\left|Q_{\phi}\right|^{2}\right),
$$


where $\left|Q_{\phi}\right|^{2}=\sum_{i, j} Q_{\phi, i j}^{2}$. If $\lambda^{2}$ achieves equality in (21), then there exists a real function $f$ such that

$$
T_{00}=2\left(\lambda^{2}-\left|Q_{\phi}\right|^{2}\right)+|f||\phi|^{2}, \quad \text { and } \quad T_{0 i}=f\left\langle\phi, e^{0} \cdot e^{i} \cdot \phi\right\rangle .
$$

Moreover, $\pm \operatorname{Tr}\left(\left.h\right|_{\partial M}\right)=H^{\partial M}$ on $\partial M$.

Remark 2. As in Remark 1, inequality (21) improves (19).

Under the regular conformal transformation $e^{2 u} \widetilde{g}$ with $d u\left(e_{r}\right)=0$, identity (15) becomes

$$
\begin{aligned}
\int_{M}|\overline{\widetilde{D}} \bar{\psi}|_{\bar{g}}^{2} v_{\bar{g}}= & \int_{M}\left(|\overline{\widetilde{\nabla}} \bar{\psi}|_{\bar{g}}^{2}+\frac{1}{2}\left\langle\bar{\psi},\left(\bar{T}_{\overline{0} \overline{0}}+\bar{T}_{\overline{0} \bar{i}} \overline{e^{0}} \overline{-} \cdot e^{i} \cdot\right) \bar{\psi}\right\rangle_{\bar{g}}\right) v_{\bar{g}} \\
& -\int_{\partial M} e^{-u}\left\langle\phi, e^{r} \cdot D^{\partial M} \phi\right\rangle * e^{r} \\
& -\frac{1}{2} \int_{\partial M} e^{-u}\left\langle\phi,\left(H^{\partial M}-\operatorname{Tr}\left(\left.h\right|_{\partial M}\right) e^{r} \cdot e^{0} \cdot\right) \phi\right\rangle * e^{r} \\
& +\frac{1}{2} \int_{\partial M} e^{-u}\left\langle\phi, h_{a r} e^{0} \cdot e^{a} \cdot \phi\right\rangle * e^{r}
\end{aligned}
$$

from which we deduce the following:

Theorem 6. Under the same conditions as Theorem 1, let $\lambda$ be a nonzero eigenvalue of the Dirac-Witten operator associated with an eigenspinor $\phi$. If $M$ has boundary $\partial M$ which is a future or past apparent horizon, moreover, $\mathcal{U}$ is nonempty and there is a positive eigenfunction $U$ of $L$ such that $U^{\frac{4}{n-2}}=$ $e^{2 u}$ for some $u \in \mathcal{U}$, then under the local boundary condition (16), we have

$$
\begin{gathered}
\lambda^{2} \geq \frac{n}{4(n-1)} \mu_{1}, \\
\lambda^{2} \geq \frac{1}{4} \mu_{1}+\inf _{M}\left|Q_{\phi}\right|^{2},
\end{gathered}
$$

where $\mu_{1}$ is the first eigenvalue of $L$ under the boundary condition $d U\left(e_{r}\right)=0$ on $\partial M$.

\section{References.}

[B] H. Baum, Spin-Strukturen und Dirac-Operatoren über pseudo-Riemannsche Mannigfaltigkeiten, Teubner-Verlag, Stuttgart/Leipzig, Band 41 (1981). 
[FS] S. Farinelli, G. Schwarz, On the spectrum of the Dirac operator under boundary conditions, J. Geom. Phys. 28, 67-84 (1998).

[F] T. Friedrich, Der erste Eigenwert des Dirac-Operators einer kompakten Riemannschen Mannifaltigkeit nicht negativer Skalarkrümmung, Math. Nach. 97, 117-146 (1980).

[GHHP] G. Gibbons, S. Hawking, G. Horowitz, M. Perry, Positive mass theorems for black holes, Commun. Math. Phys. 88, 295-308 (1983).

[He1] M. Herzlich, A Penrose-like inequality for the mass of Riemannian asymptotically flat manifolds, Commun. Math. Phys. 188, 121-133 (1998).

[He2] M. Herzlich, The positive mass theorem for black holes revisited, J. Geom. Phys. 26, 97-111 (1998).

[Hi1] O. Hijazi, A conformal lower bound for the smallest eigenvalue of the Dirac operator and Killing spinors, Commun. Math. Phys. 104, 151-162 (1986).

[Hi2] O. Hijazi, Première valeur propre de l'opérateur de Dirac et nombre de Yamabe, C. R. Acad. Sci. Paris 313, 865-868 (1991).

[Hi3] O. Hijazi, Lower bounds for the eigenvalues of the Dirac operator, J. Geom. Phys. 16, 27-38 (1995).

[HZ] O. Hijazi, X. Zhang, Lower bounds for the eigenvalues of Dirac operator, Part I. The hypersurface Dirac operators, Ann. Glob. Anal. Geom. 19, 355-376 (2001).

[HMZ] O. Hijazi, S. Montiel, X. Zhang, Eigenvalues of the Dirac Operator on Manifolds with Boundary, to appear in Commun. Math. Phys.

[LM] H.B. Lawson, M.L. Michelsohn, Spin Geometry, Princeton Math. Series, vol. 38, Princeton University Press, 1989.

[L] A. Lichnerowicz, Spineurs harmoniques, C.R. Acad. Sci. Paris 257, Série I, 7-9 (1963).

[M] B. Morel, Eigenvalue Estimates for the Dirac-Schrödinger Operators, Journ. Geom. Phys. 38 , 1-18 (2000).

[PT] T. Parker, C. Taubes, On Witten's proof of the positive energy theorem, Commun. Math. Phys. 84, 223-238 (1982). 
[W] E. Witten, A new proof of the positive energy theorem, Commun. Math. Phys. 80, 381-402 (1981).

[Z1] X. Zhang, Positive mass conjecture for five-dimensional Lorentzian manifolds, J. Math. Phys. 40, 3540-3552 (1999).

[Z2] X. Zhang, Positive mass theorem for hypersurface in 5-dimensional Lorentzian manifolds, Comm. Anal. Geom. 8, 635-652 (2000).

[Z3] X. Zhang, Lower bounds for eigenvalues of hypersurface Dirac operators, Math. Res. Lett. 5, 199-210 (1998); A remark on: Lower bounds for eigenvalues of hypersurface Dirac operators, Math. Res. Lett. 6, 465-466 (1999).

Oussama Hijazi

INSTITUT ÉLIE CARTAN

Université Henri Poincaré, NANCY I

B.P. 239, 54506 Vandeuvre-Lès-Nancy Cedex, France

hijazi@iecn.u-nancy.fr

XiaO Zhang

INSTITUTE OF MATHEMATICS

Academy of Mathematics and System Sciences

Chinese Academy of Sciences, Beijing 100080, P.R. China

xzhang@math08. math.ac.cn

ReCeived July 8, 2002. 